\title{
Particle-based simulations of powder coating in additive manufacturing sug- gest increase in powder bed roughness with coating speed
}

\author{
Eric J. R. Parteli ${ }^{1, \star}$ and Thorsten Pöschel ${ }^{2, \star \star}$ \\ ${ }^{1}$ University of Cologne, Cologne, Germany \\ ${ }^{2}$ University of Erlangen-Nuremberg, Erlangen, Germany
}

\begin{abstract}
We have developed the first particle-based numerical tool to simulate the coating of powder particles in additive manufacturing devices. Our Discrete Element Method considers realistic particle shapes and incorporates attractive interaction (van-der-Waals) forces between the particles. From simulations of powder coating using a roller as coating device, we find that the surface roughness of the powder bed scales with the square of coating speed. Moreover, we find that using fine, highly polydisperse powders may lead to larger powder bed roughness, compared to process simulations using coarser powders, due to the formation of agglomerates resulting from cohesive forces.
\end{abstract}

\section{Introduction}

Additive manufacturing (AM), which consists of building parts by successively melting powder layers by means of a laser or electron beam, has been pointed out as one of the pilars of the third industrial revolution. In particular, parts of nearly arbitrarily complex geometries can be efficiently produced without the waste of material involved in conventional machining processes [1-5].

However, to achieve mass production a quantitative understanding of the effect of powder particle shape, material properties and powder coating speed on the packing characteristics of the deposited powder layer is required. Since it is difficult to achieve this understanding by experiments alone, a particle-based tool for the simulation of the coating process, which allows for the process investigation under different powder systems and process parameters, is required. This numerical tool should account for a realistic description of inter-particle forces, particle size distributions and complex geometric shapes of the powder particles, which play major role for static and dynamic characteristics of granular systems. Here we develop such a numerical tool, based on the Discrete Element Method (DEM), and apply it for the first time to simulate powder coating using a roller as coating device. The results of our simulations show a suprising dependence of the powder bed packing behavior on particle size distributions, and suggest that increasing the coating speed may cause decrease in part quality. As we will show, DEM simulations prove useful to shed light on properties of the powder transport that are challenging to investigate in experiments.

\footnotetext{
^e-mail: eric.parteli@uni-koeln.de

${ }^{\star}$ e-mail: thorsten.poeschel@fau.de
}

\section{Numerical experiments}

The first step to achieve reliable numerical simulations of the powder application in AM is the accurate representation of the complex geometric shapes of the particles. This representation is accomplished here using the multisphere method, which consists of building clumps of spherical particles to model the target complex shape (see e.g. Refs. [6-9]). Fig. 1 shows some examples where the target shapes were obtained from light microscope images of commercially available PA12 powder as described in detail in Ref. [10].

The translational and rotational motion of the multisphere particles are dictated by the forces on the constituent spheres, that is gravity, inter-particle forces and forces between particles and the walls of the device. Given the small particle sizes involved in the process $(1-100 \mu \mathrm{m}$, see Fig. 1), attractive interaction forces must be modeled and incorporated in the DEM. In Ref. [11] we have developed a particle-based model which takes into account contact forces, as well as inter-particle attractive interactions. The contact forces are modeled by applying a modified Cundall and Strack model [12] which considers elastic and dissipative forces in the normal collision direction as well as in the tangential direction (that is, frictional forces) [1315]. Recently, we have extended this modeling to incorporate attractive particle interaction forces $[10,11]$. Our improved DEM model thus accounts for both adhesion at contact and non-bonded van der Waals forces, following the models in Refs. [16, 17]. As shown in Ref. [11], simulation predictions for the solid fraction of different types of powder covering a broad range of particle size distributions relevant for AM process agreed quantitatively with experimental results. 


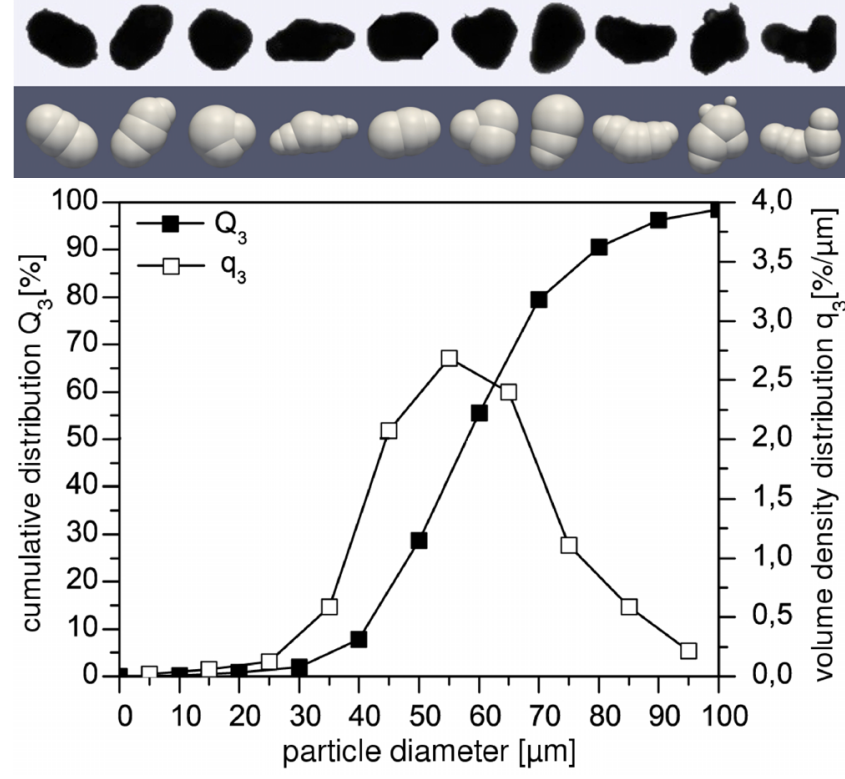

Figure 1. The upper figure shows light microscope images of PA12 powder (top) along with the corresponding model particles (bottom), while the graph shows the particle size distribution.

We have implemented the inter-particle interaction model in the DEM solver of Ref. [18] (LIGGGHTS), as this solver includes an interface to CAD programs from which we can reproduce the complex geometries of the AM devices. In the simulations, these geometries are interpreted as (dynamic) boundary conditions for the particles, whereas the force model described above has been adapted and implemented to compute particle-wall interactions (considering one of the interaction partner of infinite mass and radius). Further extensions of the DEM solver were made to incorporate the model of Ref. [8] for the correct computation of inertia tensor of the multisphere particles.

Simulations are performed with 5761 complex particles, which are modeled with the multisphere method, with the shapes shown in Fig. 1 and material parameters listed in Tab. 1 (see Ref. [10] for an in-depth discussion of the model parameters). In total, 24,771 constituent spheres are involved. Initially, the volume on both sides of the part is filled with powder particles, whereas the surface of the part is free of powder. The powder is then transported by the roller onto the part, represented by the green obstacle Fig. 2, and approximated with the multisphere method. In the simulations, the part has length (in the transport direction) of about $1 \mathrm{~mm}$.

\section{Results and discussion}

Fig. 2 shows a simulation snapshot indicating the main components of our DEM model for powder application in AM. The roller moves in positive $x$ direction with translational velocity $V_{R}$ thereby rotating counter-clockwise with a nominal rotational velocity $\omega_{R} \approx 165 \mathrm{rpm}$. While we did not find significant dependence of the results presented
Table 1. Numerical values of the parameters used in the simulations [10].

\begin{tabular}{lcl}
\hline parameter & symbol & value \\
\hline particle material density & & $1000 \mathrm{~kg} / \mathrm{m}^{3}$ \\
Young's modulus & $Y$ & $2.3 \times 10^{7} \mathrm{~Pa}$ \\
Poisson's ratio & $v$ & 0.40 \\
Coulomb's friction coefficient & $\mu$ & 0.50 \\
surface energy density & $\gamma$ & $0.1 \mathrm{~mJ} / \mathrm{m}^{2}$ \\
Hamaker constant & $A_{H}$ & $0.2 \times 10^{-21} \mathrm{~J}$ \\
\hline
\end{tabular}
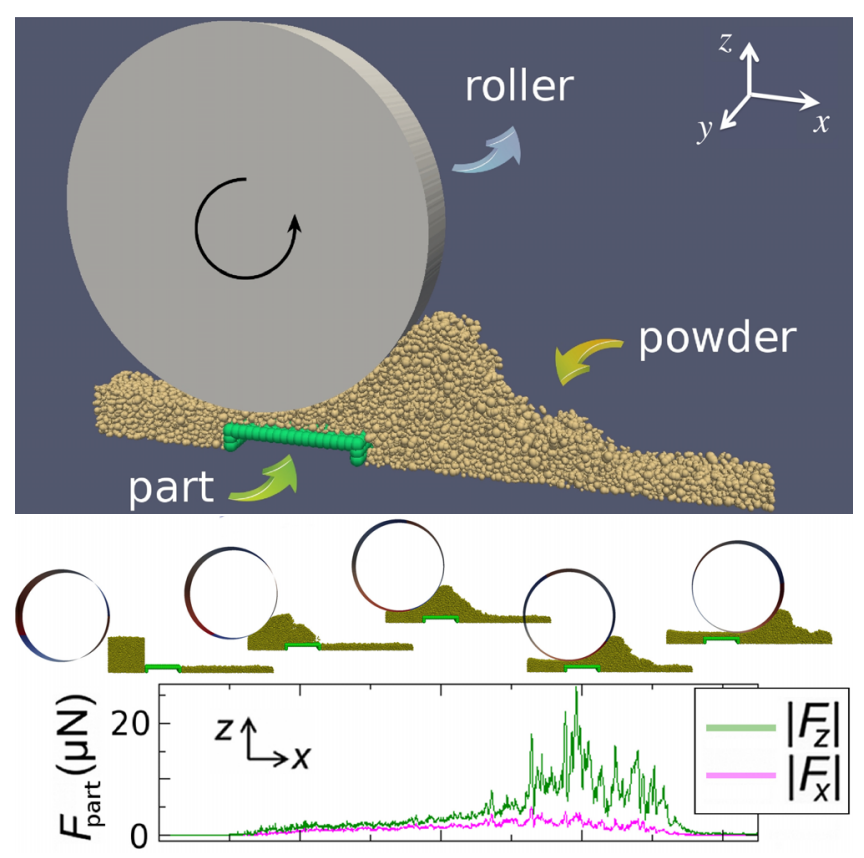

Figure 2. On top we see one snapshot of the DEM simulation while on the bottom we see the evolution of the vertical and longitudinal components of the total force on the part as the roller moves to the right (corresponding position of the roller is indicated by the snapsthos on top of the plot). The part has length $1 \mathrm{~mm}$ and $0.4 \mathrm{~mm}$ in $x$ and $y$ directions, respectively, while periodic boundary condition in $y$ direction is applied.

here on $\omega_{R}$ (see next Section for further discussion), we find that the packing behavior of the applied powder depends strongly on $V_{R}$, as we will discuss below.

We started our investigation by analyzing the evolution of the stress exerted by the granular particles transported by the roller on the part (see Fig. 1. The study of the forces within the granular system and its interactions with the device's walls and part is important as future modeling will focus on breakage and particle deformation. It is well known that stresses in granular systems are characterized by force chains which display complex dynamical behavior as the granular material is subject to external shearing or compaction. We have found (see Ref. [10]) that stress distribution within the granular bulk varies strongly over time. A fingerprint of this variation is the behavior of the total force on the part shown in the plot of Fig. 1. We see strong fluctuations in the total force that reach more than an order of magnitude, both in the vertical and in the 
horizontal direction, as the roller moves in the transport direction.

Moreover, we see that both components $F_{x}$ and $F_{z}$ nearly vanish when the roller has covered $50 \%$ of the part's length (note that time in the plot is indicated by the position of the roller relative to the bed in the upper snapshots). This result indicates the time instant from which the lowermost granular layer in the powder bed deposited on the part becomes nearly shielded from the stress due to the roller motion. However, we expect very different behavior for the plot in Fig. 2 if load in the vertical direction is exerted by the roller on the powder bed. Because this load is typically applied in experimental conditions, it constitutes one important extension to our model, whereas its effects on the total force on the part and the characteristics of force chains in the system are matter of ongoing research (first results will appear soon [20]).

Moreover, the transport dynamics largely affects the packing behavior of the powder particles deposited on the powder bed. To investigate this behavior, and since it is desired to enhance production speed while obtaining flat, densely packed powder beds, we calculated the roughness of the powder bed as a function of the roller's velocity $V_{R}$. To quantitatively characterize this roughness, we divided the powder bed on the part in 4 longitudinal slices of equal width (in $y$ direction) and computed the mean standard deviation of the maximal powder bed height $h(x)$ for all slices (where $x$ is the transport direction). The result is shown by the blue squares in Fig. 3. We see a clear increase of the bed roughness - which we call $\delta$ - with the roller's translational velocity $V_{R}$. In particular, we find that the expression

$$
\delta=a+b\left|V_{R}\right|^{2}
$$

fits well the simulation results thereby leading to $a \approx$ $6.05 \mu \mathrm{m}$ and $b \approx 3.9 \times 10^{-4} \mu \mathrm{m}$. Although this result remains to be confirmed experimentally, there is experimental evidence for increase in the roughness of powder lines deposited (poured) by a nozzle as a result of increase in coating speed.

We thus addressed the question of how particle size distribution affects the powder roughness. To investigate this issue, we modified the size distribution shown in the plot of Fig. 1 by removing all particles with diameter smaller than $60 \mu$ (but keeping the same total mass of powder particles in the numerical experiment).

The result for surface roughness $\delta$ as a function of $V_{R}$ is denoted by the circles in Fig. 3. We see that $\delta$ increases with $V_{R}$ as it does for the original size distribution, but overall values of $\delta$ obtained using the modified (coarser) powder are smaller. This is suprising result if we consider the well-known effect that deposition of highly polydisperse granular systems leads to higher solid fractions than for nearly monodisperse systems, as small particles fill the pores between larger particles. However, we can understand our results by noting that attractive inter-particle forces (adhesion and non-bonded van der Waals) lead to the formation of large agglomerates in the powder associated with the blue squares in Fig. 3, due to presence of very

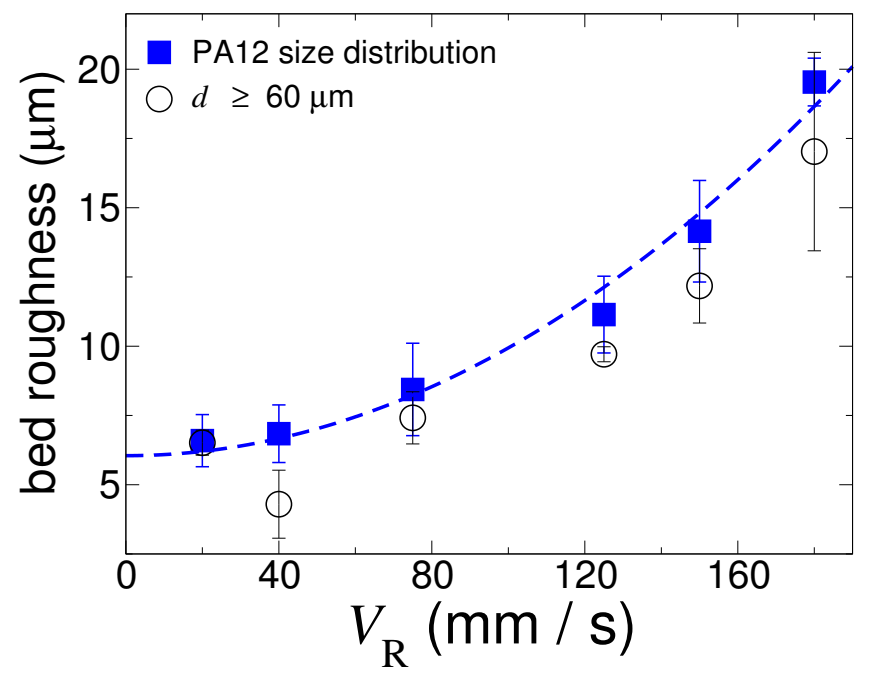

Figure 3. Roughness of the deposited powder layer as a function of the translational velocity of the roller for constant rotational velocity of $165 \mathrm{rpm}$.

small particles $<30 \mu \mathrm{m}$. For such particles, gravitational forces are largely compensated by cohesive forces, so that such small particles are transported as larger, irregular clumps or coated on the surface of much larger particles. In the modified powder size distribution, the phenomenon of agglomerate formation is less pronounced as van der Waals forces become less efficient in keeping agglomerates stable when particle size increases beyond $60 \mu \mathrm{m}$ (see Refs. [11] for a discussion on the effect of polydispersity in the solid fraction of fine powders and Ref. [10] for a more detailed analysis of the deposited powder bed in AM).

We have thus found that a way to decrease the roughness of deposited powder beds in additive manufacturing is to modify the particle size distribution so that smallest particles are filtered out from the system. This surprising result - which could be exploited to improve the quality of powder beds in AM without necessity of changing process parameters - should be now verified in experiments. Moreover, we should remark that the solid fraction may be substantially improved if vertical load is exerted by the roller on the powder bed, or when the device is subject to vibrations, which remains to be investigated.

\section{Conclusions and outlook}

We have presented a particle-based numerical tool for the simulation of powder application in additive manufacturing (AM) devices. This tool accounts for a realistic description of complex particle shapes, inter-particle forces and particle size distributions of powder systems of different materials used in AM. By applying our tool to investigate the coating process using the roller as coating device, we have found a surprising result, that is the roughness of the applied powder bed increases with the coating speed. This result, which is consequence of the cohesive interparticle forces leading to large particle agglomerates, suggests that faster production speed may not be a good op- 
tion in mass production since low solid fraction and large bed roughness may negatively influence part quality. We refer the reader to Ref. [10] for further discussions on our results as well as for a more detailed presentation of our DEM model and numerical experiments. It would be interesting to perform systematic experiments using different roller translational velocities to confirm the predictions from our simulations.

Our numerical tool provides a means to investigate particle-based processes in AM that are difficult to be studied experimentally, such as stress distribution within the powder bulk, on the part and on the coating device. We have seen that such stress distributions are characterized by force chains and large stress fluctuations that are characteristics of granular materials [19]. However, the interparticle force model should be now extended to incorporate electrostatic interactions and tribocharging, which are still poorly understood but may largely affect the transport process depending upon the powder material and process parameters.

Moreover, several questions and challenges constitute matter of ongoing [20] and future work. For instance, how do vibrations applied to the device affect the packing properties of the bed? How does the packing density depend on particle material properties or shape? What is the effect of the load of the coating system on the powder bed on the relation between coating speed and surface roughness? In particular, we have found that the rotational velocity of the roller does not play a significant role for the solid fraction of the deposited powder, but does this result change if load is applied?

We have shown how DEM simulations may help to find the answers to such questions. Following the aforementioned model improvements, our research thus advances with the vision to achieve a numerical tool that can reliably complement and largely replace systematic powder coating experiments, as well as derive strategies to optimize the production process.

\section{Acknowledgements}

We thank the Institute for Polymer Technology and the Institute for Particle Technology of the University of Erlangen-Nuremberg for the light microscope images and particle size distribution of Fig. 1, the German Research Foundation (DFG) for funding through the Cluster of Excellence "Engineering of Advanced Materials", ZISC, FPS, the Collaborative Research Center SFB814, and grant PO472/20-2. We gratefully acknowledge the computing time granted by the John von Neumann Institute for Computing (NIC) and provided on the supercomputer JUROPA at Jülich Supercomputing Centre (JSC).

\section{References}

[1] I. Campbell, D. Bourell and I. Gibson, Rapid Prototyp. J. 18, 255 (2012).

[2] R.D.Goodridge, C.J.Tuck and R.J.M.Hague, Prog. Mater. Sci. 57, 229 (2012).

[3] P. Heinl, A. Rottmair, C. Körner and R.F. Singer, Adv. Eng. Mater. 9, 360 (2007).

[4] B. Wendel, D. Rietzel, F. Kühlein, R. Feulner, G. Hülder and E. Schmachtenberg, Macromol. Mater. Eng. 293, 799 (2008).

[5] T. Stichel, T. Laumer, T. Baumüller, P. Amend and M. Schmidt, In: Proceedings of the Polymer Processing Society 29th Annual Meeting - PPS-29, Nuremberg, Germany, 2013.

[6] T. Pöschel and V. Buchholtz, Phys. Rev. Lett. 71, 3964 (1993).

[7] J. A. C. Gallas and S. Sokołowski, Int. J. Mod. Phys. 7, 2037-2046 (1993).

[8] E. J. R. Parteli, AIP Conf. Proc. 1542, 185-188 (2013).

[9] H. Pourtavakoli, E. J. R. Parteli and T. Pöschel, New J. Phys. 18, 073048 (2016).

[10] E. J. R. Parteli and T. Pöschel, Powder Technology 288, 96-102 (2016).

[11] E. J. R. Parteli, J. Schmidt, C. Blümel, K.-E. Wirth, W. Peukert and T. Pöschel, Scientific Reports 4, 6227 (2014).

[12] P.A. Cundall, O.D.L. Strack, Geotechnique 29, 47 (1979).

[13] H. Kruggel-Emden, E. Simsek, S. Rickelt, S. Wirtz and V. Scherer, Powder Technol. 171, 157 (2007).

[14] H.P. Zhang and H.A. Makse, Phys. Rev. E 72, 011301 (2005).

[15] T. Pöschel and T. Schwager, Computational Granular Dynamics (Springer, Heidelberg, 2005).

[16] H.C. Hamaker, Physica 4, 1058 (1937).

[17] K.L. Johnson, K. Kendall and A.D. Roberts, Proc. R. Soc. Lond. A Math. Phys. Sci. 324, 301 (1971).

[18] C. Kloss, C. Goniva, A. Hager, S. Amberger and S. Pirker, Prog. Comput. Fluid Dy. 12, 264 (2012). www. liggghts.com

[19] R.C. Hidalgo, H.J. Herrmann, F. Kun, E.J.R. Parteli, in: F. Mallamace, H. Stanley (Eds.), The Physics of Complex Systems (IOS Press, Amsterdam 2004), pp. 153-171.

[20] D. Schiochet Nasato, T. Pöschel and E.J.R. Parteli, Proc. ICAT (accepted, 2016).

[21] A.B. Yu, J. Bridgwater and A. Burbidge, Powder Technol. 92, 185 (1997).

[22] C.H. Liu, S.R. Nagel, D.A. Schecter, S.N. Coppersmith, S. Majumdar, O. Narayan and T.A. Witten, Science 269, 513 (1995). 